\title{
Molecular Simulation of Silica/Surfactant Self-assembly in the Synthesis of Periodic Mesoporous Silicas
}

\author{
Miguel Jorge ${ }^{*, *}$ José R. B. Gomes ${ }^{\dagger}$, M. Natália D. S. Cordeiro ${ }^{\ddagger}$ and Nigel A. Seaton ${ }^{*, \S}$ \\ ${ }^{¥}$ REQUIMTE and ${ }^{\dagger}$ CIQ-UP, Faculty of Science, University of Porto, R. Campo Alegre 687, 4169-007 Porto, Portugal \\ ${ }^{\S}$ Institute for Materials and Processes, School of Engineering and Electronics, University of Edinburgh, King's \\ Buildings, Mayfield Road, Edinburgh EH9 3JL, U.K. \\ RECEIVED DATE (automatically inserted by publisher); miguel.jorge@fc.up.pt; n.seaton@ed.ac.uk
}

Periodic mesoporous silicas (PMSs), discovered in the early $90 \mathrm{~s}^{1}$, are important materials due to their wide variety of applications ${ }^{2}$, but also due to their fundamental scientific interest. The latter stems from their unusual structures (amorphous walls and regular pore structures) and from the templated process by which they are synthesized. Indeed, the final structure of the material is intimately linked to the mesostructure of the liquidcrystal phase formed in the precursor silica/surfactant solution prior to precipitation of the solid ${ }^{3}$. The synthesis process is rather complex, involving the interplay of phase equilibrium, chemical reactions and hydrophobic effects. There is not yet a good understanding of the synthesis mechanism for this class of materials. It is perhaps surprising that only a few theoretical studies have addressed this problem ${ }^{4-6}$. These studies were based on macroscopic or coarse-grained models and have given qualitative insights into some features of PMS synthesis.

The material resulting from a given process depends strongly on the interactions and conditions at the early stages of the synthesis. A crucial stage is the formation of a so-called silicatropic liquid-crystal phase, consisting of surfactant micelles surrounded by silicate molecules ${ }^{7}$. Identification of the process by which this phase forms will not only shed light on the synthesis mechanism of PMS, but will also pave the way for a priori design strategies for these materials. In this paper, we examine the early stages of the synthesis of PMS by molecular dynamics (MD) simulation using realistic atomistic models. We are able to probe the molecular-level phenomena controlling the self-assembly of silica/surfactant mesostructures, in a way that has not been achieved in previous theoretical work ${ }^{4-6}$. We focus on MCM-41, the most widely used and studied PMS material ${ }^{8}$. In particular, we have chosen to study the synthesis process that starts from a solution of decyltrimethylammonium bromide (DeTAB), at a concentration of $0.85 \mathrm{M}$, and a monomeric silica source, at a surfactant/silica ratio of 1 , under alkaline $\mathrm{pH}=11$. These conditions do not represent the "ideal" experimental synthesis (which would start from a longer-chained surfactant cetyltrimethylammonium bromide - at a lower concentration and at a lower surfactant/silica ratio). However, our hypothetical solution would experimentally yield an MCM-41 solid ${ }^{1}$, while the relatively short surfactant makes the system computationally tractable. Thus, we are able to simulate the self-assembly process using detailed atomistic models, during sufficient time to observe the formation of micellar aggregates, and using systems that are large enough to be statistically significant.

To study the effect of silica on surfactant self-assembly, we compare two solutions: an aqueous solution of DeTAB, without silica; and a solution of DeTA ${ }^{+}$and silicic acid monomers, meant to represent an early stage of the synthesis process, in which no silica condensation has yet occurred. The relative proportion of neutral and anionic silica monomers reflects acid-base equilibrium at a $\mathrm{pH}$ of 11 . We carried out MD simulations using GROMACS $^{9}$ in the $N p T$ ensemble, at $T=298 \mathrm{~K}$ and $p=1$ bar with a time step of 2 fs. The simulations used 3-D periodic cubic boxes, starting from random initial configurations. The force field includes bending and torsional terms, Lennard-Jones (L-J) interactions and Coulomb electrostatics. Water molecules were modeled by the rigid SPC/E potential ${ }^{10}$. A united-atom potential was used for DeTA ${ }^{+}$, with parameters for the head group taken from Jorgensen and $\mathrm{GaO}^{11}$ and for the aliphatic tail taken from Smit et $a l^{12}$. The intramolecular potential constants and the L-J parameters for silicates were taken from the work of Pereira et $a l .{ }^{13}$. Their potential was developed for neutral silicates, so we carried out independent DFT calculations ${ }^{14}$ to compute charges for both neutral and anionic silicic acid monomers. Full details of the potential parameters are given in the supporting information.

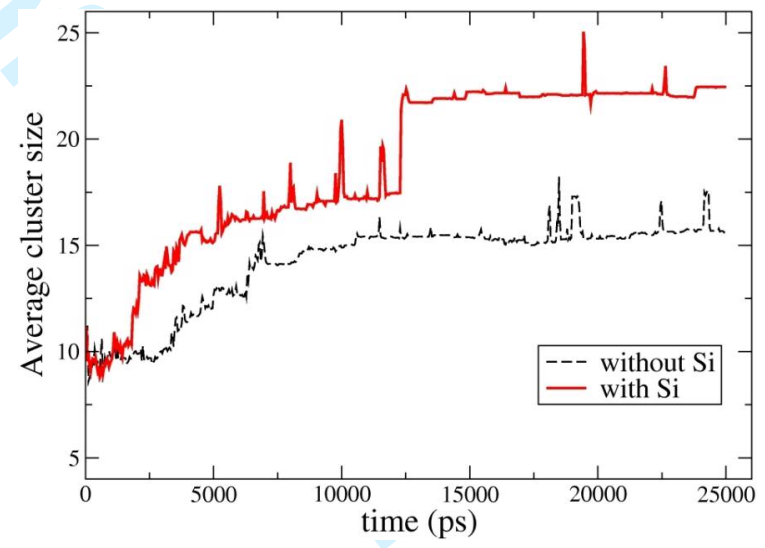

Figure 1. Plot of the mass-average cluster size as a function of simulation time.

In Figure 1 we plot the evolution of the mass-average cluster size. After a quick initial period of formation of small aggregates (up to $\mathrm{t}=2 \mathrm{~ns}$ ), in which the two solutions behave similarly, the curves start to deviate from each other. After about $15 \mathrm{~ns}$, the solution with silica shows a significantly higher average cluster size. This difference remains virtually unchanged until the end of the runs. Surfactant self-assembly is typically slow, and has probably not reached equilibrium in the $25 \mathrm{~ns}$ duration of these simulations. Nevertheless, the fast dynamics associated with the short-chain surfactant allows us to observe important processes during the early stages of self-assembly, such as monomer exchange, micelle dissolution and micelle fusion (e.g., the large step in the silica system at $12.5 \mathrm{~ns}$ is due to the fusion of two 
medium-sized aggregates). Closer examination of the micellar structures reveals other differences. In the DeTAB solution, all micelles have similar sizes, between 10 and 20 surfactants. In contrast, in the silica/DeTAB solution micelles of very different sizes are formed (between 9 and 37 surfactants). This can be clearly seen in Figure $2 b$, where there is a rather large micellar aggregate near the lower left corner of the snapshot.

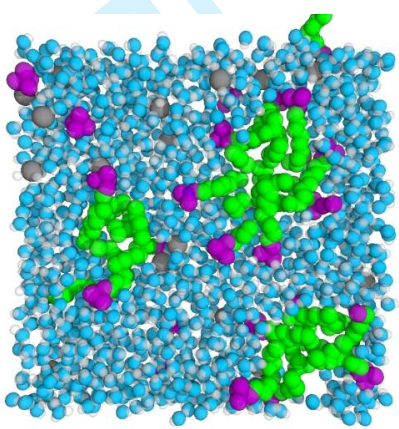

a) without $\mathrm{Si}$

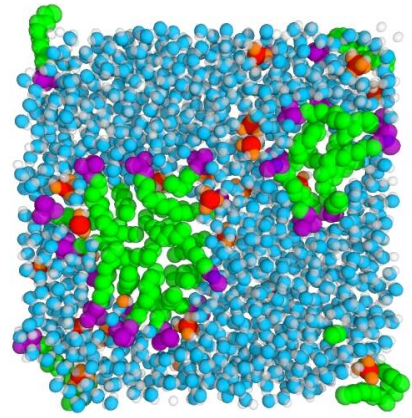

b) with $\mathrm{Si}$
Figure 2. Snapshots of a cross-section of the simulation box for the solutions with silica (right) and without silica (left) at the end of the simulation runs. Tail atoms are represented by green spheres, head atoms are purple, water oxygens are blue, silicon atoms are red, silicate oxygens are orange and hydrogens are white transparent spheres.

The formation of these large micelles in the presence of silica can be better understood if we examine their structure more closely. In Figure 3, we plot the density profile of groups of atoms with respect to the center of mass (COM) of the micelle, averaged over all micelles and the last $10 \mathrm{~ns}$ of simulation.

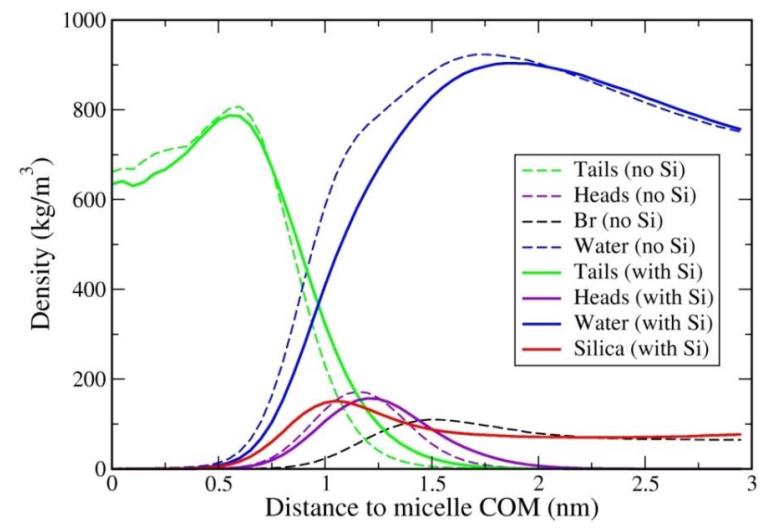

Figure 3. Plot of the average density profiles as a function of distance from the micelle center of mass, for solutions with and without silica.

Looking first at the DeTAB solution, we can see that the core of the micelle is composed exclusively of tail atoms. A relatively broad peak corresponding to head atoms marks the surface of the micelle. Water molecules are able to protrude to some extent into the micelle, approximately up to the location of the peak in tail density. Finally, a diffuse layer of bromide counterions is present at about $0.4 \mathrm{~nm}$ from the head-atom density peak. This structure of DeTAB micelles is consistent with previous experimental ${ }^{15}$ and single-micelle simulation works ${ }^{16}$. Comparing it with the silica/DeTAB solution, we can see that the overall structure is similar (the density profiles are slightly shifted because the micelles are, on average, larger), with one crucial difference - the silica anions are located much closer to the center of the micelle than the bromide ions. Indeed, the peak due to silica is located within the head-group layer. Another important difference is in the number of bound ions; in the DeTAB solution, there are on average 0.88 bromide ions per surfactant on the micelle surface, but this number rises to 1.16 for silica anions. This suggests a very strong attractive interaction between the surfactant head groups and the silicates. The presence of anionic silicates very close to the head groups counteracts their positive charges and diffuses the repulsion between them. This decreases the preferred curvature of the surface and favors the formation of larger aggregates ${ }^{17}$. The same phenomenon is known experimentally to be responsible for sphere-to-rod transitions in micellar systems ${ }^{18}$, seen in later stages of MCM-41 synthesis ${ }^{2}$. Even though we have not observed the formation of cylindrical micelles in our simulations (all micelles are slightly prolate ellipsoids), it is possible that this would occur after much longer simulation times.

The strong interaction between surfactants and silicates is responsible for a substantial increase in the local concentration of silica at the surface of the micelle. This creates an environment that shifts the equilibrium of the silica polymerization reaction to the product side, favoring the formation of larger silicate molecules. Preliminary results of ongoing simulations suggest that these larger oligomers are even more effective than monomers at producing larger surfactant micelles. Thus, we are clearly in the presence of a co-operative process - silicates promote the formation of large surfactant aggregates; these raise the local silica concentration, inducing the formation of more highly condensed silicates; these, in turn, favor even larger micelles. Our simulations shed light on the formation of silica/surfactant mesostructures, which have, until now, remained rather obscure. It is likely that these structures are important intermediates in the synthesis of periodic mesoporous silica materials. Efforts to confirm this are already underway.

Acknowledgement. Partial funding for this work was provided by the HPC-Europa programme, funded under the European Commission's Research Infrastructures activity of the Structuring the European Research Area programme, contract number RII3CT-2003-506079.

Supporting Information Available: Details of the force field parameters used in the molecular dynamics simulations. This material is available free of charge via the Internet at http://pubs.acs.org.

References

(1) Beck, J. S.; Vartuli, J. C.; Roth, W. J.; Leonowicz, M. E.; Kresge, C. T.; Schmitt, K. D.; Chu, C. T. W.; Olson, D. H.; Sheppard, E. W.; McCullen, S. B.; Higgins, J. B.; Schlenker, J. L. J. Am. Chem. Soc. 1992, 114,10834

(2) Ying, J. Y.; Mehnert, C. P.; Wong, M. S. Angew. Chem. Int. Ed. 1999, 38, 56.

(3) Raman, N. K.; Anderson, M. T.; Brinker, C. J. Chem. Mater. 1996, 8 , 1682 .

(4) Bhattacharya, A.; Mahanti, S. D. J. Phys.: Condens. Matter 2001, 13 1413.

(5) Siperstein, F. R.; Gubbins, K. E. Langmuir 2003, 19, 2049.

(6) Gov, N.; Borukhov, I.; Goldfarb, D. Langmuir 2006, 22, 605.

(7) Firouzi, A.; Atef, F.; Oertli, A. G.; Stucky, G. D.; Chmelka, B. F. J. Am Chem. Soc., 1997, 119, 3596.

(8) Selvam, P.; Bhatia, S. K.; Sonwane, C. G. Ind. Eng. Chem. Res. 2001, 40 , 3237.

(9) Berendsen, H. J. C.; van der Spoel, D.; van Drunen, R. Comp. Phys. Сотm. 1995, 91, 43.

(10) Berendsen, H. J. C.; Grigera, J. R.; Straatsma, T. P. J. Phys. Chem. 1987 $91,6269$.

(11) Jorgensen, W. L.; Gao, J. J. Phys. Chem. 1986, 90, 2174

(12) Smit, B.; Karaborni, S.; Siepmann, J. I. J. Chem. Phys. 1995, 102, 2126

(13) Pereira, J. C. G.; Catlow, C. R. A.; Price, G. D. J. Phys. Chem. A 2002, $106,130$.

(14) Gomes, J. R. B.; Cordeiro, M. N. D. S.; Jorge, M. submitted to J. Phys. Chem..

(15) Buchner, R.; Baar, C.; Fernandez, P.; Schrödle, S.; Kunz, W. J. Mol. Liq. $\mathbf{2 0 0 5}, 118,179$.

(16) Böcker, J.; Brickmann, J.; Bopp, P. J. Phys. Chem. 1994, 98, 712.

(17) Israelachvili, J. N. Intermolecular and Surface Forces; Academic Press: London, 1992.

(18) Lin, Z.; Cai, J. J.; Scriven, L. E.; Davis, H. T. J. Phys. Chem. 1994, 98 , 5984. 
Understanding the synthesis of periodic mesoporous silica (PMS) is crucial for a more efficient use of these materials and is a necessary first step towards a rational design strategy for the templated synthesis of porous solids. In this paper, the early stages of the synthesis process of PMS materials are simulated directly by molecular dynamics, using realistic atomistic models. It is the first time that such computationally demanding calculations have been attempted. By comparing the selfassembly of cationic surfactants in the presence and absence of silicates, we are able to show that silica promotes the formation of larger aggregates than in a simple surfactant/water solution. The formation of these larger micelles is explained by a strong interaction of the silicate molecules with the surfactant head groups. This strong interaction increases the local concentration of silica at the surface of the micelles, which induces the formation of more condensed silicate species. The surfactant/silica structures observed here are potentially important intermediates in PMS synthesis. 